\title{
Vaccine Refusal from the Family Doctors' Perspective: What has Changed with the COVID-19 Pandemic?
}

\author{
(D) Elif Okşan Çalıkoglu \\ Department of Public Health, Ataturk University, Erzurum, Turkey
}

\section{ABSTRACT}

Objectives: This study aimed to determine society's tendency to vaccination from the views of family physicians during the COVID-19 pandemic.

Methods: The population of the study was family physicians working in primary care and the data collection was done in November 2019 and December 2020 in the Erzurum province of Turkey. In 2019 and 2020, the total number of family physicians working in Erzurum city were 135 and 134, respectively. The participation rates in both study periods were 120 doctors (around $88.9-89.5 \%$ response rate).

Results: The medians of the physicians' durations in the profession in 2019 and 2020 were 8.0 (4.0-11.0) years and 8.0 (3.0-12.0) years, respectively $(\mathrm{p}=0.990)$. The proportion of doctors who perceived social media as the reason for vaccination refusal increased from $56(46.7 \%)$ to $92(76.7 \%)$ within one year $(p<0.001)$. Besides, $22(18.3 \%)$ of the participants, who claimed that the patient prefers catching the disease instead of being vaccinated in 2019 , had increased to $45(37.5 \%)$ in 2020 ( $p<0.001)$. Furthermore, $103(85.8 \%)$ of the participants reported that adult vaccination rates have increased with the COVID-19 pandemic. Also, the proportion of doctors, who takes a signed refusal instead of convincing the anti-vaxxers, had increased to $96(80.0 \%)$ from $73(60.8 \%)(p=0.001)$.

Please cite this article as: Çalıkoglu EO. Vaccine Refusal from the Family Doctors' Perspective: What has Changed with the COVID-19 Pandemic? Anatol J Family Med 2021;4(3):218-225.

Address for correspondence: Dr. Elif Okşan Çalıkoglu. Department of Public Health, Ataturk University, Erzurum, Turkey

Phone: +90 5053143348

E-mail:

calikogluoksan@yahoo.com

Received Date: 16.03.2021

Accepted Date: 14.06.2021

Published online: 29.12.2021

(C) Copyright 2021 by Anatolian Journal of Family Medicine -

Available online at www.anatoljfm.org

OPEN ACCESS

\begin{abstract}
Conclusion: The COVID-19 pandemic has significantly influenced the views of family physicians on the impact of social media on vaccine refusal. Considering the increasing social media activity during the pandemic process, accurate information activities on vaccination should be planned.
\end{abstract}

Keywords: COVID-19, family physicians, vaccination refusal, Turkey

\section{INTRODUCTION}

Vaccines are one of the most effective tools used to protect the population. ${ }^{[1]}$ As proven by modern medical practices, vaccination is crucial to prevent contagious infections, thereby preventing deaths or permanent sequelae caused by these diseases. ${ }^{[2]}$ Besides, vaccination provides herd immunity as well as individual immunity. As the number of vaccinated individuals in the community increases, the possibility of contact of the unvaccinated individuals with the agent and the disease's frequency in that society decrease. ${ }^{[3]}$ According to the World Health Organization (WHO) report, global immunization prevents $2-3$ million deaths a year. ${ }^{[4]}$

With the emergence of vaccine refusal and hesitation as a concept in recent decades, the frequency of vaccine refusal has increased worldwide. ${ }^{[5]}$ Perhaps for this reason, vaccination rates have decreased in some regions, and the prevalence of vaccine-preventable diseases has increased. ${ }^{[6]}$

Healthcare professionals are seen as the most trusted advisors in dealing with vaccine hesitations, having the power to influence vaccination decisions. ${ }^{[7]}$ However, it was also stated that 
clinicians themselves may be adversely affected by vaccine hesitations. ${ }^{[8]}$ In a UK study, unvaccinated healthcare professionals were more likely to agree with patients' negative vaccination decisions, while vaccinated healthcare professionals believed they should recommend the vaccine. ${ }^{[9]}$

On January 30, the WHO classified the COVID-19 epidemic as an international public health emergency. Soon, COVID-19 cases spread rapidly and were seen in many countries. Finally, it was reported as a pandemic on March 11, $2020 .{ }^{[10]}$ The high spread rate of COVID-19 scared and kept people isolated and negatively impacted healthcare utilization. ${ }^{[11]}$ During a pandemic, family physicians accomplish tasks such as reinforcing public health messages, managing patients at home, identifying patients in need of hospital care, and continuing vaccination. ${ }^{[12]}$

The hypothesis of the study is, that the concepts around vaccination may be affected by the COVID-19 pandemic. Therefore, it is aimed to determine the society's vaccination tendencies from the views of healthcare professionals during this period.

\section{METHOD}

A repeated cross-sectional study was conducted. Data collection was done in November 2019 and December 2020 in the Erzurum province of Turkey. The population of the study was family physicians working in primary care during the study dates. Located in Eastern Anatolia, Erzurum is one of the coldest cities in Turkey, with 1900 meters (6233 feet) altitude. The population of Erzurum was 762.848 people in $2019 .{ }^{[13]}$ Since the inclusion of the whole population was targeted, no sample size was calculated. In 2019 and 2020, the total number of family physicians working in Erzurum city were 135 and 134, respectively. The participation rates in both study periods were 120 doctors (around 88.9-89.5\% response rate). Flow diagram of the study is shown in Figure 1.

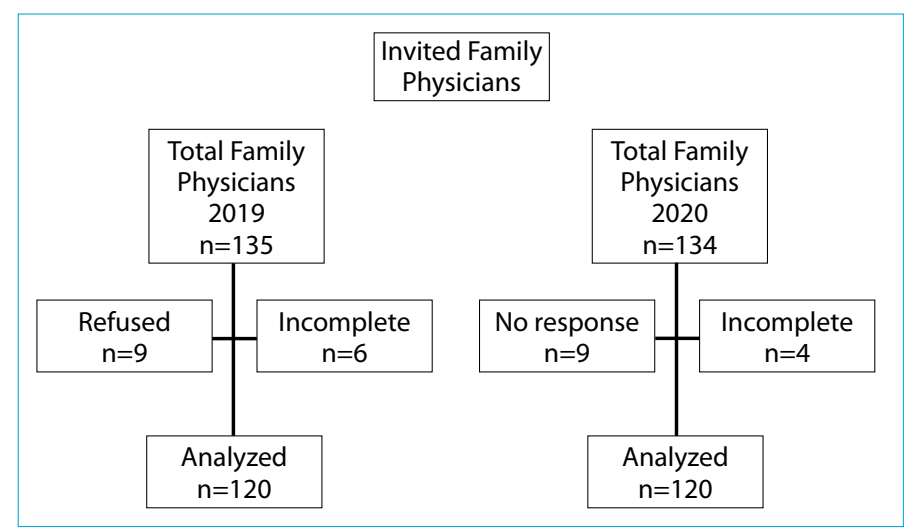

Figure 1. Flow diagram of the study.
Using the study's keywords, the primary author conducted a detailed literature search followed by three team discussions. Another team discussion with an expert panel consisting of pediatricians, family physicians, public health specialists, and infectious diseases specialists was performed to modify the study questionnaire to include COVID-19-related items. The initial study data were collected with the questionnaire (11 items) handed over to the doctors and collected back one week later. Data for the repeated study in 2020 were obtained online using Google Forms (42 items). The primary outcome variable of the study was "What are the factors affecting families in deciding to refuse vaccines?" Other study questions were "What is your approach when you face vaccine refusal?" and "What do you think are the reasons for vaccine refusal?" Other items in 2020 queried participants' views related to the COVID-19 pandemic and SARS-CoV2 vaccine. Age, duration of the profession, and gender were independent variables. The Cronbach's alpha value of the 31 -items about COVID-19 in 2020 was 0.767.

The Statistical Package for the Social Sciences software program (SPSS for Windows, Ver. 25.0, Chicago, IL, USA) was used for statistical analysis. Data were presented as median and 25th-75th percentage for numerical variables and frequency and percentage for categorical data. The suitability of variables to normal distribution was evaluated using the Kolmogorov-Smirnov test. Categorical variables were compared via the Chi-square test, while numerical variables were compared using the Mann-Whitney $U$ test. The statistical significance threshold was considered as $p<0.05$.

\section{RESULTS}

A total of 240 data points were analyzed as the sum of two consecutive years in total, where the first 120 (50.0\%) came from 2019 while the last 120 (50.0\%) was 2020's data. The medians of the attendees' ages in 2019 and 2020 were 36.0 (30.0-43.0) years and 37.0 (29.0-45.0) years, respectively $(p=0.901)$. The medians of the participants' durations in the profession in 2019 and 2020 were 8.0 (4.0-11.0) years and $8.0(3.0-12.0)$ years, respectively ( $p=0.990)$. Views of the family physicians before and after the COVID-19 pandemic are summarized in Table 1.

According to 50 (41.7\%) of the participants, staying at home during the pandemic decreased childhood vaccinations. Additionally, $96(80.0 \%)$ declared that they would recommend the COVID-19 vaccine to the population. On the other hand, while 25 (20.8\%) thought childhood vaccination rates increased with the pandemic, 77 (64.2\%) considered it did not. The rate of those who had a flu vaccine was $62(51.7 \%)$. Additionally, 35 (29.2\%) of respondents 
Table 1. Views of the family physicians before and after the COVID-19 pandemic

\begin{tabular}{|c|c|c|c|c|}
\hline & & & $x^{2}$ & $\mathbf{p}$ \\
\hline & $\begin{array}{c}2019 \\
(n=120)\end{array}$ & $\begin{array}{c}2020 \\
(n=120)\end{array}$ & & \\
\hline Gender & & & & \\
\hline Male & $66(55.0)$ & $71(59.2)$ & 0.425 & 0.514 \\
\hline Female & $54(45.0)$ & $49(40.8)$ & & \\
\hline What is you & & & & \\
\hline It is a per & & & & \\
\hline Yes & $20(16.7)$ & $10(8.3)$ & 3.810 & 0.051 \\
\hline No & $100(83.3)$ & 110 (91.7) & & \\
\hline I ask the & & & & \\
\hline Yes & $96(80.0)$ & $73(60.8)$ & 10.581 & 0.001 \\
\hline No & $24(20.0)$ & $47(39.2)$ & & \\
\hline I submit & & & & \\
\hline Yes & $68(56.7)$ & $63(52.5)$ & 0.420 & 0.517 \\
\hline No & $52(43.3)$ & $57(47.5)$ & & \\
\hline I ensure t & & & & \\
\hline Yes & $5(4.2)$ & $2(1.7)$ & 1.324 & 0.250 \\
\hline No & $115(95.8)$ & $118(98.3)$ & & \\
\hline What do yo & & & & \\
\hline Commer & & & & \\
\hline Yes & $53(44.2)$ & $47(39.2)$ & 0.617 & 0.432 \\
\hline No & $67(55.8)$ & $73(60.8)$ & & \\
\hline Vaccines & & & & \\
\hline Yes & $47(39.2)$ & $48(40.0)$ & 0.017 & 0.895 \\
\hline No & $73(60.8)$ & $72(60.0)$ & & \\
\hline Religious & & & & \\
\hline Yes & $87(72.5)$ & $78(65.0)$ & 1.571 & 0.210 \\
\hline No & $33(27.5)$ & $42(35.0)$ & & \\
\hline Believing & & & & \\
\hline Yes & 45 (37.5) & $22(18.3)$ & 10.953 & 0.001 \\
\hline No & $75(62.5)$ & $98(81.7)$ & & \\
\hline Believing & & & & \\
\hline Yes & $77(64.2)$ & $64(53.3)$ & 2.906 & 0.088 \\
\hline No & $43(35.8)$ & $56(46.7)$ & & \\
\hline Believing & & & & \\
\hline Yes & $82(68.3)$ & $68(56.7)$ & 3.484 & 0.062 \\
\hline No & $38(31.7)$ & $52(43.3)$ & & \\
\hline Side effe & & & & \\
\hline Yes & $63(52.5)$ & $42(35.0)$ & 7.467 & 0.006 \\
\hline No & $57(47.5)$ & $78(65.0)$ & & \\
\hline What are th & & & & \\
\hline Social me & & & & \\
\hline Yes & $92(76.7)$ & $56(46.7)$ & 22.844 & $<0.001$ \\
\hline No & $28(23.3)$ & $64(53.3)$ & & \\
\hline
\end{tabular}




\section{Table 1. CONT.}

\begin{tabular}{|c|c|c|}
\hline & & \multirow[t]{2}{*}{$x$} \\
\hline 2019 & 2020 & \\
\hline$(n=120)$ & $(n=120)$ & \\
\hline
\end{tabular}

Healthcare workers with anti-vaccine attitudes

Yes

No

Anti-vaccine groups on social media

Yes

No

Friends and relatives

Yes

No
57 (47.5)

$63(52.5)$

88 (73.3)

$32(26.7)$

68 (56.7)

$52(43.3)$
$108(90.0)$

50.444

$<0.001$

$12(10.0)$

39 (32.5)

40.153

$<0.001$

$81(67.5)$

87 (72.5)

6.576

0.010

$33(27.5)$

Data is presented as $\mathrm{n}(\%)$.

Chi-Square test.

had COVID-19, and 26 (21.7\%) believed they would easily recover if they caught it. The views of the family physicians about COVID-19 and its vaccine are summarized in Table 2.

\section{DISCUSSION}

The COVID-19 pandemic has significantly influenced family physicians' views on the impact of social media on vaccine refusal over time. Besides, the rate of participants who think that "the idea, being sick is preferable than vaccination, causes vaccination refusal in society" has increased. Moreover, the proportion of those who wanted to get the applicant to sign a vaccine refusal increased significantly. In addition, the number of participants who thought that unwanted side effects after vaccination was one of the reasons for vaccine refusal increased significantly. According to $41.7 \%$ of the participants, the decline in the number of applications to health institutions due to the pandemic had an impacted decreased childhood vaccinations. Besides, $80 \%$ declared that they would recommend the COVID-19 vaccine to the population. However, only $65.9 \%$ of them thought of getting the COVID-19 vaccine. According to $85.8 \%$ of the participants, vaccination rates of older person have increased with the pandemic. On the other hand, while $20.8 \%$ thought childhood vaccination rates increased with the pandemic, $64.2 \%$ considered the opposite. Furthermore, $36.7 \%$ stated that the COVID-19 vaccine rejection rates would be high in Erzurum, and 39.2\% expected low vaccine refusals in Turkey. Besides, $87.5 \%$ of doctors believed that there will be people among the registered population who will refuse the COVID-19 vaccine. The rate of those who had the flu vaccine was $51.7 \%$. Finally, $29.2 \%$ of respondents had COVID-19, and $21.7 \%$ believed they would easily recover if they catch it.

The WHO recommends each country taking steps to understand vaccine hesitation at the local level, to adequately respond to these needs and concerns that differ depending on the cultural, social, and personal beliefs of a particular region. ${ }^{[14]}$ However, in many countries, there is no surveillance program for vaccine refusal or hesitation. Hence, most of the information we have available is retrospective and anecdotal. ${ }^{[15]}$

The rate of vaccine hesitation or refusal is relatively low in most societies. ${ }^{[16]}$ Therefore, when conducting a study on this subject, it is necessary to include many people to reach a sufficient sample, which in turn, requires high cost and labor. On the other hand, since they are in constant contact with thousands of people, healthcare professionals are good reflectors for assessing vaccine refusal or hesitation. In the current health system of Turkey, routine vaccination is only possible after registering with a family physician. Also, they play a critical role in helping patients gain a positive perspective on vaccination. For instance, knowing the benefits of immunization with the information that can be given by healthcare professionals is a useful strategy to prevent vaccine hesitation or refusal. ${ }^{[17]}$ Considering all these factors together, family physicians seem as practical resources to collect information on public tendencies.

Despite the enormous socio-economic impact of the COVID-19 pandemic, it was determined that not everyone would want to get the SARS-CoV-2 vaccine. ${ }^{[17]}$ Taking into account that these doctors could play a key role in this issue, the rate of family physicians who would recommend 


\section{Table 2. The views of the family physicians about COVID-19} and its vaccine

n (\%)

The COVID-19 vaccination will completely end the pandemic in the society

Yes

45 (37.5)

No

Not sure

$47(39.2)$

$28(23.3)$

The decrease in referrals to health institutions due to the pandemic has reduced childhood vaccinations

$$
\text { Yes }
$$

Not sure

All vaccines, including the COVID-19, should be obligatory

Yes

No

Not sure

The Expanded Vaccination Program place liability on Family Physicians

Yes

No

Not sure

In Turkey, only smallpox vaccine is legally required

Yes

No

Not sure

Vaccination will disable the implementation of other preventive measures (mask, distance, hygiene) in the pandemic

Yes

No

Not sure

COVID-19 vaccination will be free of charge in Turkey

Yes

No

Not sure

To ensure herd immunity, $100 \%$ vaccination rates should be reached in the society

Yes

No

Not sure

Refusal of the COVID-19 vaccine will not impose liability on the family physician

Yes

No

Not sure

$69(57.5)$

$1(0.8)$

$60(50.0)$

$38(31.7)$

22 (18.3)

28 (23.3)

$74(61.7)$

$18(15.0)$

$68(56.7)$

18 (15.0)

34 (28.3)

$69(57.5)$

39 (32.5)

$12(10.0)$

$103(85.9)$

$1(0.8)$

$16(13.3)$

$44(36.7)$

68 (56.7)

$8(6.6)$

\section{Table 2. CONT.}

n (\%)

Would you recommend the COVID-19 vaccine to the population you are responsible for?

Yes

$96(80.0)$

No

7 (5.8)

Not sure

$17(14.2)$

In my registered population, some people will refuse the COVID-19 vaccine

Yes

105 (87.5)

No

$12(10.0)$

$3(2.5)$

Not sure

$101(84.2)$

$6(5.0)$

$13(10.8)$

Not sure

Vaccinating healthcare professionals will increase community compliance with the COVID-19 vaccination

Yes

$96(80.0)$

No

$11(9.2)$

Not sure

$13(10.8)$

COVID-19 is an occupational disease for healthcare professionals

Yes

$113(94.2)$

No

$6(5.0)$

$1(0.8)$

It is the right decision to first administer the COVID-19 vaccine to healthcare professionals

Yes

88 (73.3)

No

$29(24.2)$

Not sure

$3(2.5)$

Would you volunteer for phase studies of COVID-19 vaccines under development?

Yes

29 (24.2)

No

$73(60.8)$

Not sure

$18(15.0)$

Are you going to get vaccinated against the COVID-19 vaccine?

Yes

79 (65.9)

No

$13(10.8)$

Not sure

$28(23.3)$

Would you prefer a locally produced vaccine?

Yes

$76(63.3)$

No

20 (16.7)

Not sure

24 (20.0)

Does the protection level of the vaccine affect your vaccination decision?

Yes

94 (79.0)

No

$20(16.8)$

Not sure 


\section{Table 2. CONT.}

Does the production technology of the vaccine

for COVID-19 (Inactivated vaccine, mRNA vaccine)

affect your vaccination decision?

Yes

No

Not sure

There is no need to vaccinate people who had

COVID-19

Yes

No

Not sure

COVID-19 vaccine should not be administered

to populations over the age of 65 .

Yes

No

Not sure

Babies and children should be a priority for

COVID-19 vaccination

Yes

No

Not sure

The pandemic has increased the interest of people in the community for vaccines

Yes

No

Not sure

Childhood vaccination rates have increased with the pandemic

Yes

No

Not sure

Adult vaccination rates have increased with the pandemic

Yes

No

Not sure

Only the COVID-19 vaccine should be required

by law

Yes

No

Not sure

Like the flu vaccine, the COVID-19 vaccine will

be repeated every year

Yes

No

Not sure

COVID-19 vaccine-refusal rates will be low

in Turkey.

Yes

No

Not sure
78 (65.0)

$30(25.0)$

$12(10.0)$

37 (30.8)

$64(53.4)$

$19(15.8)$

5 (4.2)

103 (85.8)

$12(10.0)$

98 (81.7)

$17(14.2)$

103 (85.8)

14 (11.7)

3 (2.5)

25 (20.8)

77 (64.2)

$18(15.0)$

103 (85.9)

$16(13.3)$

$1(0.8)$

$30(25.0)$

68 (56.7)

$22(18.3)$

73 (60.9)

16 (13.3)

$31(25.8)$

$19(15.8)$

\section{Table 2. CONT.}

\begin{tabular}{ll} 
& $\mathbf{n}(\%)$ \\
\hline $\begin{array}{l}\text { COVID-19 vaccine-refusal rates will be high } \\
\text { in Erzurum } \\
\text { Yes }\end{array}$ \\
$\quad$ No & $44(36.7)$ \\
$\quad$ Not sure & $47(39.2)$ \\
The concept of infodemic is a risk for vaccination & $29(24.1)$ \\
$\quad$ Yes & \\
$\quad$ No & $72(60.0)$ \\
Not sure & $13(10.8)$ \\
\end{tabular}

the COVID-19 vaccine in our study was considered low. Moreover, the number of doctors who stated that they will have the COVID-19 vaccine was less than this proportion. Although it is expected that some doctors may not be vaccinated because they think they already had the COVID-19 disease, these rates are not sufficient for a group serving as role models to society.

With the widespread use of the internet, most people have access to information from non-reliable sources. According to a study, more than $80 \%$ of internet users were looking for health issues, and $16 \%$ of them were related to vaccines. ${ }^{[18]}$ Also, people regularly share vaccine information on social media platforms, and the anti-vaccination movement is using social media as a tool. Besides, a growing number of people (especially young ones) get most of their news from social media. ${ }^{[19]}$ Additionally, in a case-control study conducted by Salmon et al., it was shown that individuals have difficulty determining which of the multiple sources of information on social media are reliable. ${ }^{[20]}$

It was claimed that due to social media, where messages can spread very quickly without editorial supervision, measles-mumps-rubella vaccination rates decreased, leading to an increase in measles cases. ${ }^{[21,22]}$ In line with previous studies, our study demonstrated a significant impact of social media on vaccine refusal. Moreover, the COVID-19 pandemic has amplified this effect. We think staying home during the pandemic increases the use of social media. Therefore, they are more affected than before.

It has been observed that whether the information is right or wrong does not significantly differ in terms of the speed of its spread in social media. ${ }^{[23]}$ Besides, infodemic, which has become widespread on social media during the COVID-19 pandemic, may lead to COVID-19 treatment and vaccine compliance problems. ${ }^{[23,24]}$ Consistent with the literature, most of our participants stated that infodemic 
adversely affected people, which may be attributed to the growth of social media.

Increased adult vaccination rates during the COVID-19 pandemic is one of the remarkable findings of the study. The belief among adults on vaccinations preventing the severe course of COVID-19 disease (for example, influenza vaccine) may have influenced this result. ${ }^{[25]}$ Due to the interest in adult vaccines, the Ministry of Health of Turkey changed flu vaccination priorities. ${ }^{[26]}$

In a study with primary healthcare providers in the USA, 1 in 10 healthcare providers reported that $10 \%$ of applicants' parents refused the vaccine. ${ }^{[27]}$ In our study, the proportion of doctors asking parents who refused the vaccine before and after the COVID-19 pandemic to sign a form was higher than the $31 \%$ reported in the above study. However, data from our research found that doctors tended to be more reluctant and protective during the COVID-19 pandemic. Compared to 2019, the proportion of those who sufficed with a vaccine refusal signature instead of providing detailed counseling increased significantly in 2020. The increased workload due to the pandemic or not wanting to spend too much time with the applicant to avoid contamination may have contributed to this outcome.

This study had some limitations. Although most of the repeated survey participants were the same, no matching of the responses could be done due to ethical considerations. The lack of a well-studied instrument for data collection was considered as another limitation. Also, due to the COVID-19 outbreak, the data of the second study were obtained online.

\section{CONCLUSION}

The main outcome from this study is, that the COVID-19 pandemic has significantly influenced family physicians' views on the impact of social media on vaccine refusal. Yet, the influence of the social media is not always positive in terms of increasing the vaccination numbers or introducing the benefits of the vaccination in general, instead, it is now numerically highlighted that the negative effects are also indispensable. There should be better communication channels concerning the broad benefits of immunization, and this should be part of the education programs of health professionals.

\section{Disclosures}

Peer-review: Externally peer-reviewed.

Conflict of Interest: None declared.

Ethics Committee Approval: Ethical approval was obtained from the Atatürk University Faculty of Medicine Clinical Research Ethics Committee (Approval date: Dec 17, 2020, and Approval number: B.30.2ATA.0.01.00/524).

\section{REFERENCES}

1. National Vaccine Advisory Committee. Protecting the public's health: critical functions of the Section 317 Immunization Program-a report of the National Vaccine Advisory Committee. Public Health Rep 2013;128(2):78-95.

2. McNeil MM, DeStefano F. Vaccine-associated hypersensitivity. J Allergy Clin Immunol 2018;141(2):463-72.

3. Andre FE, Booy R, Bock HL, Clemens J, Datta SK, John TJ, et al. Vaccination greatly reduces disease, disability, death and inequity worldwide. Bull World Health Organ 2008;86(2):140-6.

4. WHO. Immunization coverage 2020. Available at: https:// www.who.int/news-room/fact-sheets/detail/immunizationcoverage. Accessed Jan 7, 2021.

5. Smith TC. Vaccine Rejection and Hesitancy: A Review and Call to Action. Open Forum Infect Dis 2017;4(3):ofx146.

6. Phadke VK, Bednarczyk RA, Salmon DA, Omer SB. Association between vaccine refusal and vaccine-preventable diseases in the United States: a review of measles and pertussis. JAMA 2016;315(11):1149-58.

7. Paterson P, Meurice F, Stanberry LR, Glismann S, Rosenthal SL, Larson HJ. Vaccine hesitancy and healthcare providers. Vaccine 2016;34(52):6700-6.

8. Williams JTB, O'Leary ST, Nussbaum AM. Caring for the vaccine-hesitant family: evidence-based alternatives to dismissal. J Pediatr 2020;224:137-40.

9. Marcu A, Rubinstein H, Michie S, Yardley L. Accounting for personal and professional choices for pandemic influenza vaccination amongst English healthcare workers. Vaccine 2015;33(19):2267-72.

10. Abid K, Bari YA, Younas M, Tahir Javaid S, Imran A. Progress of COVID-19 epidemic in Pakistan. Asia Pac J Public Health 2020;32(4):154-6.

11. Zhang YN, Chen Y, Wang Y, Li F, Pender M, Wang N, et al. Reduction in healthcare services during the COVID-19 pandemic in China. BMJ Glob Health 2020;5(11):e003421.

12. Saatçı E. COVID-19 Pandemic and health professionals: Keep them alive or survive? Turkish J Fam Pract 2020;24(3):153-66.

13. von Elm E, Altman DG, Egger M. The StrengthGening the Reporting of Observational Studies in Epidemiology (STROBE) statement: uildelines for reporting observational studies. J Clin Epidemiol 2008;61:344-9.

14. Wikipedia. Erzurum; 2020. Available at: https://en.wikipedia. org/wiki/Erzurum. Accessed Nov 28, 2020.

15. WHO. Immunization, vaccines and biologicals-Improving vaccine demand and addressing hesitancy. Geneva: WHO; 2019.

16. McAteer J, Yildirim I, Chahroudi A. The VACCINES Act: Deci- 
phering vaccine hesitancy in the time of COVID-19. Clin Infect Dis 2020;71(15):703-5.

17. Dredze M, Broniatowski DA, Smith MC, Hilyard KM. Understanding vaccine refusal: why we need social media now. Am J Prev Med 2016;50(4):550-2.

18. Societal impact of vaccination : beyond individual protection. Renewed interest following COVID-19 pandemic ?. [Article in French]. Rev Med Liege 2020;75(S1):170-5.

19. Kata A. Anti-vaccine activists, Web 2.0, and the postmodern paradigm--an overview of tactics and tropes used online by the anti-vaccination movement. Vaccine 2012;30(25):3778-89.

20. Mitchell A, Gottfried J, Matsa KE. Millenials and political news. Social media-the local tv for the next generation. Washingt Pew Cent; 2015. Available at: https://www.pewresearch.org/ journalism/2015/06/01/millennials-political-news/. Accessed Dec 14, 2021.

21. Salmon DA, Moulton LH, Omer SB, DeHart MP, Stokley S, Halsey NA. Factors associated with refusal of childhood vaccines among parents of school-aged children: a case-control study. Arch Pediatr Adolesc Med 2005;159(5):470-6.

22. Burki T. Vaccine misinformation and social media. Lancet Digit Heal 2019;1(6):e258-9.

23. Puri N, Coomes EA, Haghbayan H, Gunaratne K. Social media and vaccine hesitancy: new updates for the era of COVID-19 and globalized infectious diseases. Hum Vaccin Immunother 2020;16(11):2586-93.

24. Cinelli M, Quattrociocchi W, Galeazzi A, Valensise CM, Brugnoli E, Schmidt AL, et al. The COVID-19 social media infodemic. Sci Rep 2020;10(1):16598.

25. Brennen JS, Simon F, Howard PN, Nielsen RK. Types, sources, and claims of COVID-19 misinformation. Reuters Inst 2020;7:1-3. Available at: https://reutersinstitute.politics.ox.ac. uk/types-sources-and-claims-covid-19-misinformation. Accessed Dec 13, 2021.

26. Marín-Hernández D, Schwartz RE, Nixon DF. Epidemiological evidence for association between higher influenza vaccine uptake in the elderly and lower COVID-19 deaths in Italy. J Med Virol 2021;93(1):64-5.

27. Hacaloğlu H. Why does Turkey have a flu vaccine problem?. Available at: https://www.amerikaninsesi.com/a/ turkiye-neden-grip-asisi-sorunu-yasiyor-kimlere-asi-yapilacak-/5633522.html. Accessed Jan 12, 2021.

28. Kempe A, Daley MF, McCauley MM, Crane LA, Suh CA, Kennedy $A M$, et al. Prevalence of parental concerns about childhood vaccines: the experience of primary care physicians. Am J Prev Med 2011;40(5):548-55. 